\title{
Foot Plantar Pressure Measurement Using Low Cost Force Sensitive Resistor (FSR): Feasibility Study
}

\author{
A. Jor ${ }^{1 *}$, S. Dash ${ }^{2}$, A. S. Bappy ${ }^{2}$, A. Rahman ${ }^{3}$ \\ ${ }^{1}$ Department of Leather Engineering, Khulna University of Engineering \& Technology, Khulna- \\ 9203, Bangladesh \\ ${ }^{2}$ Department of Electronics and Communication Engineering, Khulna University of Engineering \& \\ Technology, Khulna-9203, Bangladesh \\ ${ }^{3}$ Department of Industrial Engineering and Management, Khulna University of Engineering \& \\ Technology, Khulna-9203, Bangladesh
}

Received 14 March 2019, accepted in final revised form 26 May 2019

\begin{abstract}
Plantar pressure measurement is an essential and effective strategy in the assessment of foot musculoskeletal safety and footwear comfort. It is commonly applied in clinical gait analysis, sports and foot biomechanical studies. But most of the devices used for plantar pressure measurement are high cost and not readily available for the research. This study aims to present a low cost foot plantar pressure measurement system based on force sensitive resistor (FSR). The system is composed of low cost electronic instrumentation through FSR, arduino, LCD display, baseboard and a power bank. To verify the feasibility and effectiveness of the system, a linear load vs. pressure calibration test was made and a group of female participants performed static trials on heels of three different heights. Experimental results and comparative analysis showed that this system has stable performance in static measurements of plantar pressure.
\end{abstract}

Keywords: FSR; Foot; Plantar pressure measurement; Footwear; Heel.

(C) 2019 JSR Publications. ISSN: 2070-0237 (Print); 2070-0245 (Online). All rights reserved. doi: http://dx.doi.org/10.3329/jsr.v11i3.40581 J. Sci. Res. 11 (3), 311-319 (2019)

\section{Introduction}

Monitoring of plantar pressure is becoming very popular in clinical, sports, and design of footwear and rehabilitation studies. This technology can help to identify and analyze pathologies, muscular defects, postural anomalies, design of footwear, geometry of outsole and evaluate gait and standing posture [1-4]. There are some technologies that

* Corresponding author: abujorlekuet10@gmail.com 
have developed to measure foot plantar pressures but most of those devices are high cost and not available to the researchers from lower developed countries. Self-constructed plantar pressure monitoring systems have been developed by many researchers. Satio et al. developed a prototype device to monitor in-shoe plantar pressure during walking [5]. Crea et al. introduced a pressure sensitive insole for the study of plantar pressure distribution [6]. Motha et al. demonstrated an instrumental rubber insole printed with embedded pressure sensor for detection and investigation of plantar pressure [7]. Tan et al. designed and developed a plantar pressure measuring smart insole [8]. Varoto et al. described the development and application of insole sensor to monitor foot plantar pressure [9]. Recently, some commercial techniques stand out in the analysis of plantar pressure, body motion and for gait characterization. Baropodometric Platforms and Sensorized Insoles (Sensor Medica, Italy) are commercially available for the evaluation of foot plantar pressure, and patient's posture during walking and standing. The medilogic WLAN insole (Medilogic, Germany) aids in orthopaedic footwear making. The Pedar System (Novel, Germany) is a technique of monitoring dynamic pressure distribution between the foot and footwear. The F-Scan System (Tekscan, USA) provides flexible tactile resistive insole sensors to get dynamic information about foot function and gait. But most of these systems are costly and not commercially available in all countries within the research budget. Force sensitive resistor (FSR) is a low cost and frequently used for pressure measurement. Yun et al. applied FSR to evaluate the comfort of driver seat [10]. Smith et al. used FSR to improve walking in the child with cerebral palsy through triggering electrical stimulation [11]. Pawin et al. showed human's abnormal gait can be calculated by using the signal from low cost FSR through installed in the sole of a shoe [12]. Sayed et al. used FSR for the recognition of surface texture [13]. Though there are many techniques available for the detection of foot gait analysis, it needs more research to develop and make the product available commercially within low cost. The aim of this study is to describe the feasibility of low cost way for foot plantar pressure measurement in static condition using FSR. The device was made through low cost (less than 12 USD) electronic instrumentation and a data acquisition module. To verify the feasibility of the system, load vs. pressure linearity tests were realized and a group of participants performed random trials based on static activities of the system in different heel heights.

\section{Materials and Methods}

\subsection{Data acquisition module}

FSR is a sensor that senses the pressure applied on it. It is used to measure the physical pressure and weight. It converts the pressure into voltage and then detects the pressure.

The FSR consists of 2 layers as shown in Fig. 1, named sensing film and conductive polymer. The electrically conductive and non-conductive particles of sub-micrometer 
sizes form the sensing film. The conductive polymer changes the resistance in accordance with the applied force on its surface. Two surfaces are separated by a plastic spacer.

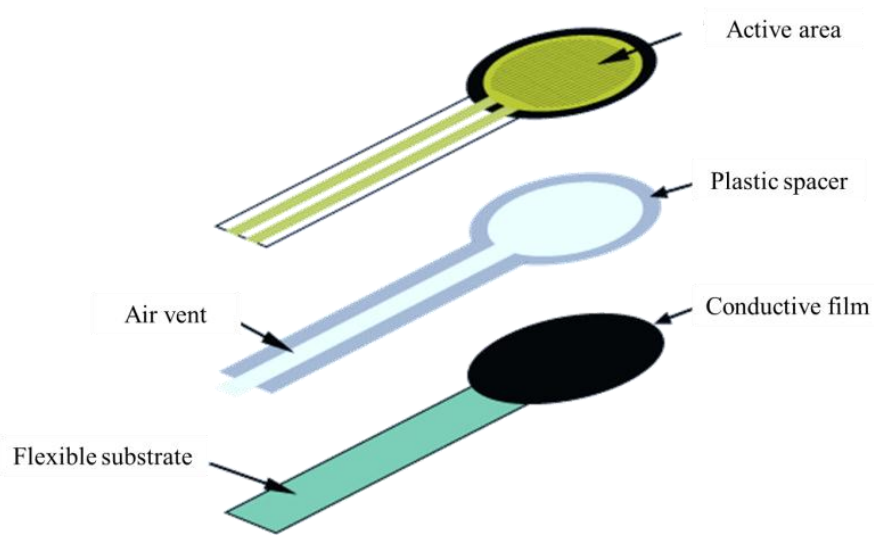

Fig. 1. Layers of Force Sensitive Resistor (FSR) [14].

One terminal of the FSR is connected to the $\mathrm{V}_{\mathrm{cc}}$ and the other is grounded through a Pull-Down resistor, $\mathrm{R}_{\mathrm{PDR}}$. $\mathrm{R}_{\mathrm{FSR}}$ is a variable FSR resistor and $\mathrm{R}_{\mathrm{PDR}}$ is a fixed resistor. Analog output voltage, $V_{o}$ is collected from the combined point of $R_{F S R}$ and $R_{P D R}$ then used as the input voltage at the analog input point.
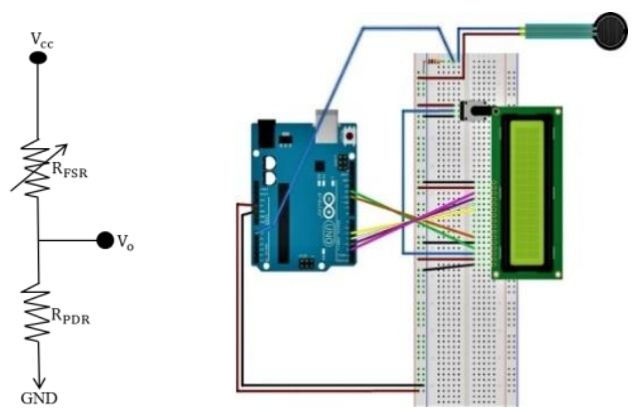

Fig. 2. Wiring diagram of FSR and LCD monitor with Arduino.

Liquid Crystal Display (LCD) has 12 pins among which 6 pins are digital pin. 4 Digital pins (pin D4 to pin D7) of LCD were connected with the digital pins (pin 4 to pin 7) of Arduino. Then the Register Select (RS) pin (pin 4) of the LCD was connected to the Arduino at pin number 1 and the Enable (EN) pin of the LCD was connected to the pin number 2 of the Arduino. Mode of the R/W pin was selected as write mode to write and send commands and data to the LCD.

The conducting resistance between the sensing film and the conductive polymer is responsible for the current conduction. Active element dots are connected with the 
conductive polymer when force is applied on the FSR surface which created more effective area for current conduction. As the more force is applied, the more contact path is created which decreases the resistance of the FSR. Output analog voltage can be represented as,

$V_{o}=\frac{R_{P D R}}{R_{P D R}+R_{F S R}} V_{c C}$

Analog output voltage follows the voltage divider rules. When forces were applied on FSR surface, the resistance $\mathrm{R}_{\mathrm{FSR}}$ was decreased. According to the voltage divider rules, voltage across the Pull-Down resistance, $\mathrm{R}_{\mathrm{PDR}}$ was increased. So the analog output voltage was increased with the increasing pressure.

It scaled the changing voltage into Newton. To do this conversion, the system was calibrated using some known weights.

\subsection{Load vs. pressure tests for FSR verification}

The sensor was selected for the experiment after being tested with known weights for the characteristic calibrated curve (Fig. 3) [15]. The applied force range was from 0 to $60 \mathrm{~kg}$. The calibrated outcomes with linearity factors of almost one and coefficients of assurance of $\mathrm{R}^{2}>0.99$ demonstrate that the estimation framework is a dependable technique to detect foot plantar pressure.

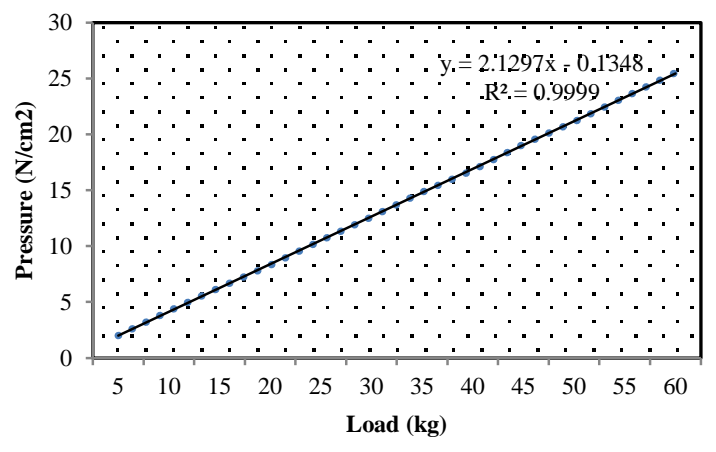

Fig. 3. Calibration curve of load vs. pressure.

\subsection{Experiment setup}

The system is consisted of FSR 406 (Interlink Electronics), arduino, LCD display, baseboard and a power bank. The instrumentation is associated with the switching circuit, and performs analog to digital conversion of voltage and resistance into pressure (Fig. 4). 


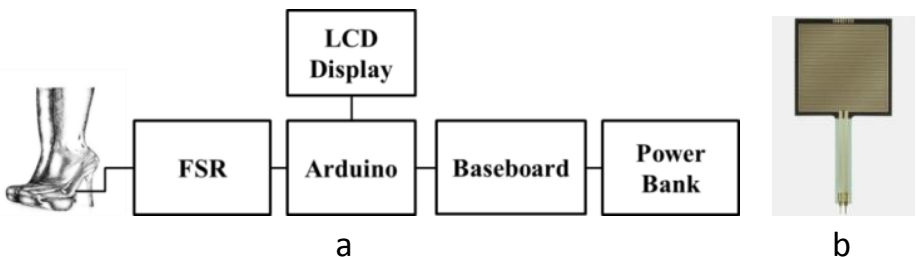

Fig. 4. (a) Block diagram of the system; (b) FSR; with $39.6 \mathrm{~mm}$ active area.

The switching circuit, including modified voltage dividers, converts resistance into voltage. Furthermore, the microcontroller provides serial data to data acquisition module that sends it to LCD display as pressure $\mathrm{N} / \mathrm{cm}^{2}$.

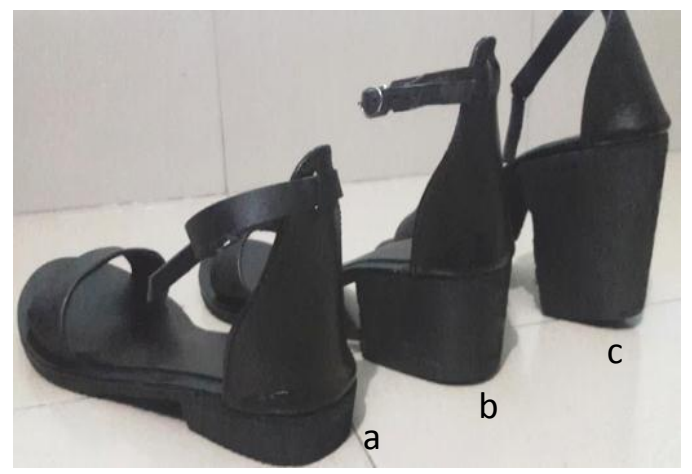

Fig. 5. High heel with three different heights; from left to right (a) flat heel, (b) medium high heel, and (c) high heel.

The high-heel female footwear's used in this study were custom made with similar construction and sole materials (Fig. 5). The height of the heel was the only difference among this footwear's and specified as: flat heel $(1 \mathrm{~cm})$, medium heel $(5 \mathrm{~cm})$, and high heel $(9 \mathrm{~cm})$.

\subsection{Foot plantar pressure measurement}

Eight normal female participants were selected for the test and data collection. The average age, height and weight of the group of participants were 21 years, $159 \mathrm{~cm}$ and 56 $\mathrm{kg}$ respectively. All participants are habituated with different types of heels.

The participants were asked to walk for $5 \mathrm{~min}$ with their regular pace. After that the FSR sensor of the system was placed beneath the foot plantar surface and on the medial forefoot area (below $1^{\text {st }}$ and $2^{\text {nd }}$ metatarsal head) between foot and insole. To make the placement of FSR in a similar way with respect to the foot bottom surface and metatarsal region, a frame was made with a pattern paper. During standing, data of plantar pressure was monitored and presented in Table 1. In the same way, the process was completed for the rest of high heel footwear with all participants. 
Table 1. peak plantar pressure on medial forefoot of three different heel heights.

\begin{tabular}{ccc}
\hline Condition & $\mathrm{n}$ & Mean \pm SD \\
\hline $1 \mathrm{~cm}$ height & 8 & $21.5 \pm 1.59$ \\
$5 \mathrm{~cm}$ height & 8 & $36.5 \pm 2.10$ \\
$9 \mathrm{~cm}$ height & 8 & $44.0 \pm 2.50$ \\
\hline
\end{tabular}

Statistical data analysis of forefoot peak plantar pressure was conducted with a statistical tool. Experimental data was compared and analyzed with some previous studies that have been done on plantar pressure at different heel heights.

\section{Results and Discussion}

The calibration curve for the verification of working ability was plotted as load versus pressure in Fig. 6. The coefficient of the determination indicated that the device was working properly. During experiment as the heel height was increased from flat to high heel, the load increased gradually. The plantar pressure was increased to $69.7 \%$ when shifted from flat to medium heel and it was raised $104 \%$ from flat to high heel footwear. With the comparison of medium to high heel it was raised to $20.5 \%$.

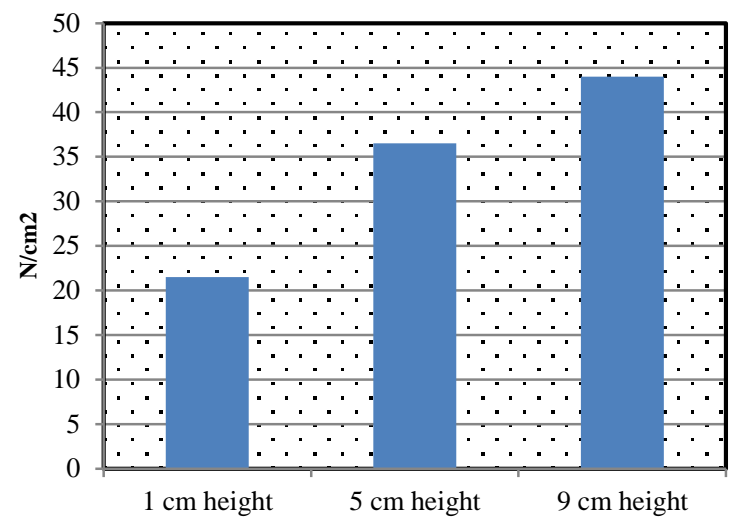

Fig. 6. Foot plantar pressure; from left to right flat heel $(1 \mathrm{~cm})$, medium heel $5 \mathrm{~cm}$, and high heel $(9$ $\mathrm{cm})$.

The obtained results were compared with the previous study of the measured plantar pressure from Cong et al. [16], Yung-Hui et al. [17] and Gu et al. [18] and is displayed in Fig. 7. 


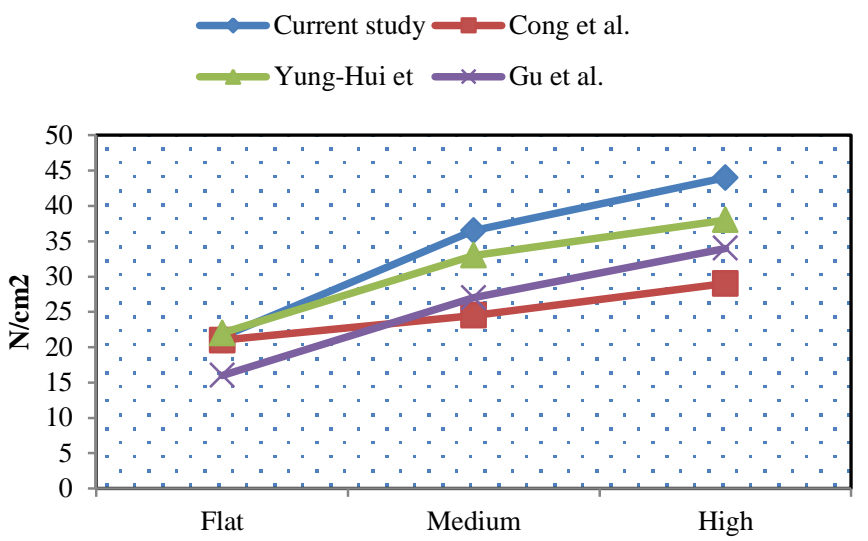

Fig. 7. Comparison of experimental data with previous studies; (a) Cong et al. [16], Yung-Hui et al. [17] and Gu et al. [18].

Cong et al. [16] studied on in shoe triaxial stresses based on different heel heights. They used three different heights $(3,5$ and $7 \mathrm{~cm})$ of ladies heel footwear and measured foot plantar pressure of ten female participants using force sensing transducer. The plantar force under $1^{\text {st }}$ and $2^{\text {nd }}$ metatarsal head increased gradually with heel height and showed $17 \%$ when shifted low heel to medium, $18.3 \%$ when medium to high heel, and $38 \%$ when shifted from low heel to high heel. Yung-Hui et al. [17] investigated the plantar pressure on foot inserts when heel height increased from flat $(1 \mathrm{~cm})$, medium $(5.1 \mathrm{~cm})$, to high heel $(7.6 \mathrm{~cm})$. They used pedar plantar pressure measurement system (Novel, Germany) to monitor pressure distribution and found a gradual change with heel height as $50 \%$ when shifted from flat to medium, $72 \%$ when compared from flat to high heel and it was $15 \%$ when shifted from medium to high heel. Gu et al. applied twelve female participants to observe foot loading on outsole area while walking in high heeled shoe. Heel with three different heights flat $(0 \mathrm{~cm})$, medium $(4.5 \mathrm{~cm})$, and high $(8.5 \mathrm{~cm})$ were used for the investigation and found remarkable change when shifted from flat to medium (40\%), medium to high $(26 \%)$, and flat to high $(112.5 \%)$. They obtained their data through the novel embed system.

The measured values from the present study were compared and analyzed through statistical tool and their respective relativity coefficients were obtained from the comparison and it was $r^{2}{ }_{1}=0.93, r_{2}{ }_{2}=0.99$, and $r^{2}{ }_{3}=0.99$ respectively. The graph $r 1, r 2$, and $\mathrm{r} 3$ were plotted in Fig. 8a-c for both the experimental and review paper. The relativity of coefficient values demonstrates that there is a good connection of the developed system with referenced studies. 

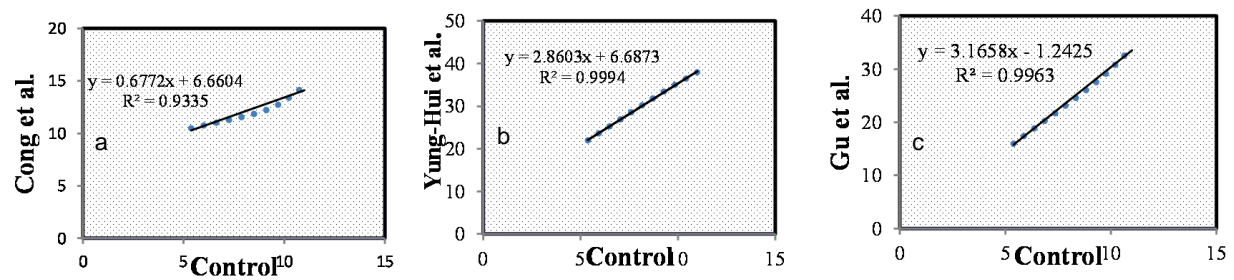

Fig. 8. Relativity coefficient graph of comparative data with previous study (a) Cong et al. [16], Yung-Hui et al. [17] and Gu et al. [18].

These results indicated that the system of plantar pressure measurement with low cost FSR is possible with acceptable accuracy. Above calibration and verification of performance by the participants indicated that the system or device was effective to monitor the static foot plantar pressure. In the future, Internet of Things (IoT) can be applied to record and observe the data in dynamic condition smoothly and remotely.

\section{Conclusion}

In this study, feasibility of FSR as a low cost plantar pressure measurement system is presented. The main features of this device are low cost, simple structure and data collection system, good plantar pressure detection and portable which makes the system applicable for laboratory research, clinical gait, and activity monitoring. Due to low cost and easy to setup, all people in general can afford to use the device in personal research and analysis. For example to monitor the plantar pressure in respect to different situation, test how much force is applied on different bone position with different types of footwear. Here, only the static condition was studied. It needs more study about how to make the device perfect for the analysis of pressure in dynamic condition.

\section{Acknowledgments}

The co-operative help from S. Payel, department of Leather Engineering (KUET) is appreciated.

\section{References}

1. M. Bellizzi, G. Rizzo, G. Bellizzi, M. Ranieri, M. Fanelli, G. Megna, and U. Procoli, Strabismus 19, 21 (2011). https://doi.org/10.3109/09273972.2010.545469

2. C. W. Kaercher, V. K. Genro, C. A. Souza, M. Alfonsin, G. Berton, and J. S. C. Filho, BMC women's health 11, 51 (2011). https://doi.org/10.1186/1472-6874-11-51

3. J. Chapman, S. Preece, B. Braunstein, A. Höhne, C. Nester, P. Brueggemann, and S. Hutchins, Clin. Biomech. 28, 679 (2013). https://doi.org/10.1016/j.clinbiomech.2013.05.005

4. J. Melvin, S. Preece, C. Nester, and D. Howard, Gait posture 40, 682 (2014). https://doi.org/10.1016/j.gaitpost.2014.07.026

5. M. Saito, K. Nakajima, C. Takano, Y. Ohta, C. Sugimoto, R. Ezoe, K. Sasaki, H. Hosaka, T. Ifukube, and S. Ino, Med. Eng. Phys. 33, 638 (2011). 
6. S. Crea, M. Donati, S. M. M. De Rossi, C. M. Oddo, and N. Vitiello, Sensors 14, 1073 (2014). https://doi.org/10.3390/s140101073

7. L. Motha, J. Kim, and W. S. Kim, Org. Electronics 23, 82 (2015). https://doi.org/10.1016/j.orgel.2015.04.020

8. A. M. Tan, F. K. Fuss, Y. Weizman, Y. Woudstra, and O. Troynikov, Procedia Technol. 20, 117 (2015). https://doi.org/10.1016/j.protcy.2015.07.020

9. R. Varoto, G. C. Oliveira, A. V. F. de Lima, M. M. Critter, and A. Cliquet Jr., In, Biodevices, pp. 207-214 (2017). DOI: 10.5220/0006246402070214

10. M. H. Yun, L. Donges, and A. Freivalds, Adv. in Ind. Ergonomics and Safety IV, 403-410 (London: Taylor and Francis, 1992).

11. B. T. Smith, D. J. Coiro, R. Finson, R. R. Betz, and J. McCarthy, IEEE Transact. Neural Syst. Rehabilit. Eng. 10, 22 (2002). https://doi.org/10.1109/TNSRE.2002.1021583

12. J. Pawin, T. Khaorapapong, and S. Chawalit, - Fourth International Workshop on Advanced Computational Intelligence (IWACI) (Wuhan, 2011), pp. 224-229. https://doi.org/10.1109/IWACI.2011.6160007

13. M. Sayed, J. C. D. Garcia, and L. Alboul - Conference Towards Autonomous Robotic Systems, (Springer, UK, 2016) pp. 288-294. https://link.springer.com/chapter/10.1007/978-3-31940379-3_30

14. F. Noya, hackaday, (2017). https://hackaday.io/project/27056-ebers/log/66516-sensor-design

15. F. Vecchi, C. Freschi, S. Micera, A. M. Sabatini, P. Dario, and R. Sacchetti - 5th Ann. Conf. of Int. FES (Country, 2000).

16. Y. Cong, J. T.-M. Cheung, A. K. Leung, and M. Zhang, J. Biomech. 44, 2267 (2011).

17. L. Yung-Hui and H. Wei-Hsien Appl. Ergonomics 36, 355 (2005). https://doi.org/10.1016/j.apergo.2004.11.001

18. Y. Gu, M. Rong, and G. Ruan, Procedia Environ. Sci. 8, 464 (2011). https://doi.org/10.1016/j.proenv.2011.10.073 